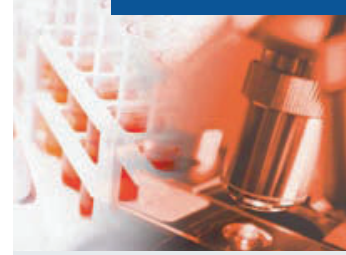

Berichte aus der Pharmaindustrie
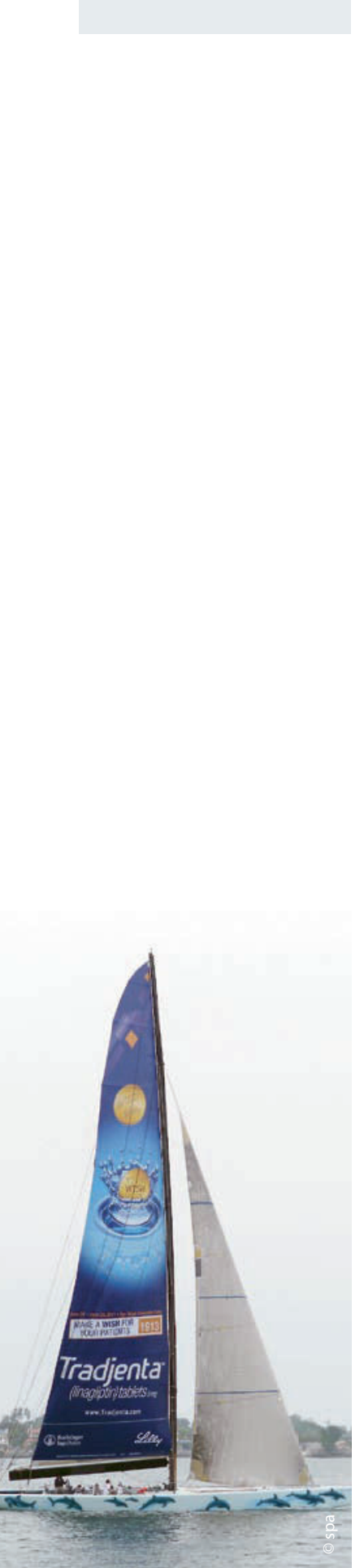

Marketing made in USA: Boot beim ADA-Kongress in San Diego

\title{
Typ-2-Diabetes
}

\section{Neuer GLP-1-Agonist wirkt additiv zu Basalinsulin}

- In zwei Phase-III-Studien des GetGoal-Studienprogrammes erwies sich der neue GLP-1-Agonist Lixisenatid additiv wirksam zu einer Behandlung mit einem Basalinsulin.

Lixisenatid (vorgesehenes Warenzeichen Lyxumia ${ }^{\circledR}$ ) ist ein neuer GLP-1-Agonist zur einmal täglichen s.c.Applikation, der von Sanofi-Aventis klinisch entwickelt wird. Mit der Zulassungseinreichung in Europa wird im 2. Halbjahr 2011 gerechnet. Beim US-Diabetes-Kongress wurden die Ergebnisse zweier 24-Wochen-Studien mit zusammen fast 800 Patienten vorgestellt, in denen Lixisenatid zusätzlich zu einem Basalinsulin für eine signifikante $\mathrm{HbA}_{1 \mathrm{c}}$-Senkung sorgte. In der GetGoal-L-Asia-Studie senkte der GLP1-Agonist das $\mathrm{HbA}_{1 \mathrm{c}}$ von einem Ausgangswert von $8,5 \%$ versus Placebo um $0,9 \% ; 36 \%$ versus $5 \%$ der Patienten erreichten $\mathrm{HbA}_{1 \mathrm{c}}$-Werte unter 7\%. Bei diesen Patienten stieg das Risiko für leichte bis mittelgradige Hypoglykämien nur dann an, wenn sie zusätzlich Sulfonylharnstoffe erhielten. In der GetGoal-L-Studie bei überwiegend kaukasischen Patienten, die als $\mathrm{Zu}$ satztherapie Basalinsulin mit oder ohne Metformin bekamen, erhöhte der GLP-1-Agonist das Risiko für Hypoglykämien nicht. Zudem nahmen die Patienten mit Lixisenatid signifikant Gewicht ab. Hauptnebenwirkung war, wie bei GLP-1-Agonisten üblich, Übelkeit in den ersten Therapiewochen.

Das klinische Entwicklungsprogramm umfasst neun Phase-III-Studien des GetGoal-Programmes mit 4300 Patienten sowie eine kardiovaskuläre Endpunktstudie (ELIXIA) mit 6000 Patienten. In letztgenannte wird placebokontrolliert und doppelblind bei Typ-2-Diabetikern nach akutem Koronarsyndrom geprüft, ob der GLP-1-Agonist Herztod, Infarkt, Schlaganfall oder instabile Angina Pectoris verhindern kann. Die Studie läuft über 3,5 Jahre.

Eine andere Studie zeigte erstmals kardioprotektive Effekte von Lixisenatid an isolierten Rattenherzen. Nach vorübergehender Ischämie konnte es die Infarktgröße um 36\% reduzieren, ohne die Hämodynamik zu beeinflussen, und besserte die myokardiale Glukoseaufnahme nach regionaler Ischämie. de

Quelle: Veranstaltung beim ADA-Kongress, 24.6.2011 in San Diego, Veranstalter: Sanofi-Aventis

\section{Neuer DDP-Hemmer mit Zusatznutzen Keine Dosisanpassung bei Leber- und Niereninsuffizienz}

- In den USA bereits zugelassen, in Europa ante portas: Linagliptin wird die Palette der inkretinbasierten Antidiabetika bereichern. Ein wichtiger praktischer Pluspunkt: Aufgrund eines von anderen DPP4-Hemmern unterschiedlichen Abbauwegs braucht die Dosierung bei Patienten mit Nieren- und Leberinsuffizienz nicht adaptiert zu werden.

Der wissenschaftliche Ausschuss der Europäischen Arzneimittelagentur hat Ende Juni eine Empfehlung für die Zulassung von Linagliptin (Tradjenta $\left.{ }^{\circledast}\right) 5 \mathrm{mg}$ Filmtabletten einmal täglich zur Therapie von erwachsenen Typ-2-Diabetikern ausgesprochen. Das haben die kooperierenden Unternehmen Boehringer Ingelheim und Lilly mitgeteilt. Die Markteinführung in Deutschland ist für September geplant. Linagliptin zielt auf das Dipeptidylpeptidase-Isoenzym 4, das kompetitiv und selektiv gehemmt wird, nicht aber andere Isoenzyme wie DPP-8 und DPP-9. DPP-4 hemmt den Abbau des Inkretins GLP-1. Dadurch wird die Sekretion von Insulin verstärkt und die von Glukagon gehemmt. Ersteres geschieht glukoseabhängig, weshalb Hypoglykämien nicht gefördert werden.

An den Zulassungsstudien für Linagliptin beteiligten sich weltweit etwa 4000 Typ-2-Diabetiker. Als wichtiger praxisrelevanter Pluspunkt erweist sich der Abbauweg von Linagliptin, die Substanz bzw. Meta- boliten werden via Fäzes ausgeschieden, brauchen also weder Niere noch Leber zur Verstoffwechselung. Wie beim ADA-Kongress erläutert wurde, ist deshalb weder bei Nieren- noch Leberinsuffizienz eine Dosisanpassung nötig, gleichgültig welches Gewicht, Geschlecht oder welche Rasse ein Patient hat.

Die Studien zur Glukosekontrolle prüften $\mathrm{HbA}_{1 \mathrm{c}}$, Nüchternblutzucker und postprandialen 2-StundenBZ bei Typ-2-Diabetikern mit einem Ausgangs$\mathrm{HbA}_{1 \mathrm{c}}$ von knapp > 8\%. Bei Monotherapie mit Linagliptin war die Glukosekontrolle signifikant besser als mit Placebo. 25\% der Patienten erreichten sogar einen $\mathrm{HbA}_{1 \mathrm{c}}<7 \%$. In Kombination mit Metformin war die Wirkungssteigerung ebenfalls statistisch signifikant und 28,3\% der Patienten erreichten das Ziel $\left(\mathrm{HbA}_{1 \mathrm{c}}<7 \%\right)$. Quer durch alle Phase -III-Studien mit allen gängigen oralen Kombinationen (außer Insulin) betrug die $\mathrm{HbA}_{1 \mathrm{c}}$-Abnahme im Mittel zwischen 0,5 und 0,7\%. Das Sicherheitsprofil gab keinen Anlass zu Bedenken. Als häufigste Nebenwirkung wurden Halsschmerzen bei über 5\% der Patienten gemeldet. Die Hypoglykämiequote betrug in Monotherapie und in Kombination mit Metformin $\leq 1 \%$.

$a u$

Quelle: Edelman SV, Basile JN. A new DPP-4 inhibitor for the treatment of adult patientes with type-2-diabetes; Präsentation beim der ADA-Kongress, San Diego, 25.6.2011. 